\title{
THE PROBLEM OF RECURRENT PEPTIC ULCER AFTER OPERATION
}

\author{
By Hermon Taylor, M.A., M.D., M.Ch., F.R.C.S. \\ Surgeon, London Hospital; Surgeon, King George Hospital, Ilford
}

\section{General Considerations}

Although little enough is known about the cause of the onset of duodenal ulceration, there is no doubt that its persistence is associated with increased acidity of the gastric juice. This is a physical character of the individual, based upon an excess of acid-secreting mucosa in the stomach.

Failure to reduce this acidity is the chief cause of post-operative ulceration at the anastomosis, since the jejunum is even more vulnerable to acid chyme than the duodenum. Thus in experimental animals gastro-jejunal ulcers can be produced by simply by-passing the alkaline juices of the duodenum into the intestine beyond the gastric anastomosis. The counterpart of this arrangement in human surgery is a gastro-enterostomy made with a Roux ' $\mathrm{Y}$ ' loop, or the addition of an entero-anastomosis to an otherwise standard operation. Both these procedures nearly always result in ulceration at the stoma and have long since been discarded in practice.

When a gastro-enterostomy is performed for duodenal ulcer, the acid juice is diverted away from the lesion and it promptly heals. The operation may, of course, result in a further erosion at the anastomosis, whereby the patient will be in worse case than before; but the ulcer in the duodenum will always heal. Radiologists, unable in some cases to demonstrate the real cause of the trouble at the stoma, and observing the old duodenal deformity, are apt to interpret this as persistence of the ulcer there. This is practically never so; only complete stenosis of the stoma will cause a reactivation of the original duodenal lesion after gastro-enterostomy.

Gastric ulcers are not generally associated with increased secretion or acidity of peptic juice, and it seems clear that this is the reason for their remarkable immunity from trouble at the anastomosis after operation. This applies not only to partial gastrectomy for gastric ulcer, but also to the very effective operation of gastro-enterostomy for 'hand-bag' deformity of the stomach with stasis, due to extensive contraction of the lesser curve.

\section{The Problem}

The incidence of recurrent ulcer after gastroenterostomy has been the subject of many exaggerated statements. There is no doubt that many patients have been cured by this operation and have, moreover, retained their full health and vigour. Nevertheless, about 30 per cent. of cases sooner or later do develop further ulcer symptoms This was first generally realized in this country in the years preceding the second world war, and led to the virtual abandonment of gastro-enterostomy in favour of partial gastrectomy. Since that time, however, it has gradually become clear that the price to be paid for a cure of ulcer dyspepsia by extensive resection of the stomach involves a diminution of general robustness and vitality, a degree of gastronomic disability and a liability to a number of indirect effects of greater or less degree. The price is not too high to avoid the certainty of another ulcer, but that is not the position in 70 per cent. of cases. A solution to the problem has been sought in the addition of vagotomy to the short-circuiting procedure, but it appears that, in general, this gives rise to rather more trouble than does a resection. Similarly, ingenious techniques to replace the excised part of the stomach by grafts of large or small intestine have not yet materially improved the results of gastrectomy.

In these circumstances it is well to remember that there are differences in the degree of hyperacidity in duodenal cases, which roughly correspond to the liability of the patients to recurrent ulcer after operation. For example, the young man with a high acidity who develops his ulcer early in life is the type most liable to stomal ulceration, particularly after gastro-enterostomy. On the other hand it is often stated that it is safe 


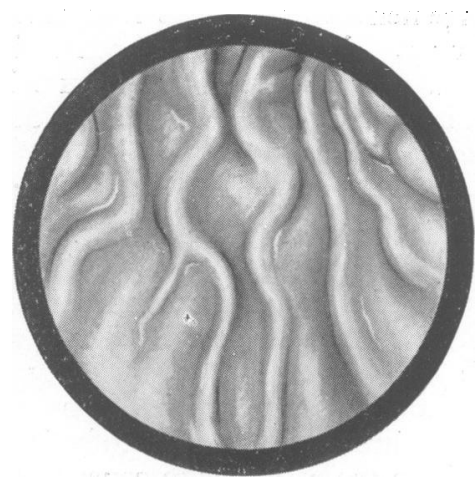

A. Normal gastric mucosa.

to carry out a gastro-enterostomy for those cases of duodenal ulcer that have gone on to stenosis. This again is broadly true, because these are longstanding cases, generally with mild symptoms and associated with only a moderate excess of acid, which involves a correspondingly reduced irritation at the stoma.

\section{Selection of Cases}

Pre-operative selection of cases from the standpoint of the degree of the hyperacidity must therefore be considered. This was much in vogue in the period between the wars. In those days, before the advent of antibiotics and non-toxic anaesthesia, partial gastrectomy was an operation involving a considerable risk to life, whereas gastro-enterostomy was not. The choice of operation was therefore most anxiously weighed, and the major procedure was only undertaken if the test meal revealed a very high acidity. The results with regard to recurrent ulceration were disappointing, chiefly perhaps because a test meal in hospital is a poor guide to the acid potential of the patient in ordinary life. Nevertheless the theory was sound, and once more becomes important now that gastrectomy, though no longer risky, has proved to be not devoid of undesirable sequelae.

It is interesting, therefore, to find that the degree of hyperacidity appears to be roughly proportional to the number and size of the folds in the gastric mucosa; the greater the surface area, the greater the acid-secreting potential of the individual. This emerged quite clearly from a gastroscopic review of Sir James Walton's follow-through series of gastro-enterostomized duodenal patients carried out by the writer I4 years ago. Those patients who had been cured were the ones with the fewer rugae, while those with stomal ulcers almost invariably displayed a considerable excess of folds. Since then new

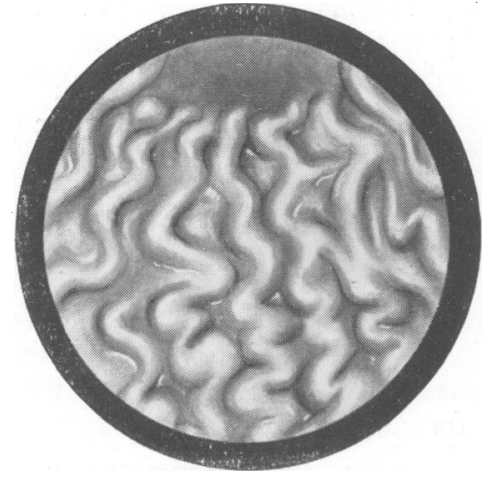

B. Hypertrophic, as in duodenal ulceration.

duodenal cases coming to operation have been segregated for gastro-enterostomy or partial gastrectomy according to the endoscopic assessment of the gastric rugosity. About half the cases have been judged suitable for gastro-enterostomy and among these about ro per cent. of cases have developed an anastomotic ulcer during the I4year period of careful follow-up. One must regard this figure as inconclusive-neither good enough to support nor bad enough to condemn this method of pre-operative selection of cases.

The follow-up of these patients has, however, emphasized an important observation, namely the extremely good health of the successful gastroenterostomy patient, compared with the mere relief of ulcer symptoms and generally only moderate well-being of the successful partial gastrectomy case.

Another point that has been brought out is that after gastro-enterostomy a patient is vulnerable to erosion at the stoma at any subsequent time, particularly during a period of stress or anxiety. Such cases should therefore be kept under supervision. They need not be restricted in diet or activity, but they should make a lifelong habit of taking two hourly snacks by day, and a prophylactic dose of antacid powder at bed-time to counteract the excess night secretion which is their special character.

\section{Symptoms}

The symptoms of gastro-jejunal ulcer are similar to those of any peptic ulcer in their time relations to meals, relief with food and alkali, and the periodicity of the attacks. Their distinctive qualities derive from their situation and the tendency to partial occlusion of the jejunum by oedema during the attacks. Thus, after gastroenterostomy, the tenderness is situated rather to the left of the mid-line at umbilical level-the site of the anastomosis-and there is a variable 
degree of vomiting of bile, which often brings relief. After gastrectomy, the stoma is usually high out of reach under the left costal margin, and there may be no tenderness at all, just a severe diffuse pain often referred to the left shoulder or to the interscapular region. These signs and symptoms are diagnostic and hardly subject to confirmation by investigation, particularly as this is often difficult and inconclusive. The presence of the stoma destroys the value of any test meal observations, while on X-ray the super-imposition of the gastric on the jejunal shadow makes it very difficult for the radiologist to demonstrate an anastomotic ulcer. He has more often to rely on the conjunction of the tenderness with the observed stoma and the presence of distortion of the jejunum in this area. Frankly positive radiological signs are the exception rather than the rule. Gastroscopy is more effective in demonstrating an ulcer at the stoma, but the deformity here may possibly prevent a view of the stoma being obtained at all. However, it is relatively easy to inspect a normal stoma, and gastroscopy is of more value in excluding an anastomotic ulcer in a doubtful case than in confirming the presence of one that is clinically obvious.

\section{Complications}

Stomal ulcers may lie actually on the old suture line, or in the jejunum nearby. In the latter event the lesion is usually in the efferent coil of jejunum and stenosis may then cause gastric retention and bilious vomiting to become the dominant symptom. Acute haemorrhage may result from erosion of a jejunal vessel, but it is not as a rule as profound as the severest forms of duodenal or gastric haemorrhage. Repeated small occult haemorrhages are more characteristic, and result in a persistent or recurrent anaemia which may, perhaps, be the only indication of the lesion. Perforation of a stomal ulcer may result in contamination of the peritoneal cavity with intestinal contents, so that the degree of the infection and toxic absorption may be more serious than with a perforated duodenal ulcer.

\section{Gastro-jejunal Colic Fistula}

The particular complication to which gastrojejunal ulcers are prone is perforation into the transverse colon, with the formation of a gastrojejuno-colic fistula. It is curious that this nearly always results in the healing of the ulcer, but the effects of the fistula may be disastrous. The obvious cases are those in which the patient either belches foul gas and vomits faecal material, or develops diarrhoea with recognizable food in the stools. A combination of these symptoms is uncommon, the fistula generally having a valve- like arrangement which causes it to work in one direction only. For this reason both barium mes and barium enema examinations must be made when it is wished to demonstrate the fistula radiologically.

The more insidious cases are those in whic the fistula is small with no immediately recogo nizable symptoms. Instead there is a graduaf development of a sprue-like syndrome in whick the patient becomes anaemic, loses weight, suffer vitamin $B$ deficiencies and passes bulky offensive stools with a high content of fat. The reason for this is the chronic low-grade infection of the small gut from the fistula, with consequent failure of absorption, especially of fat. The abdomen becomes distended and at operation there is ofte $\overline{\text {. }}$. a curious brown pigmentation of the jejunumi These patients are ill from malnutrition and operation is fraught with danger unless it is preceded by an adequate course of intestinat antibiotics. The response to these is oftent dramatic; the patient's appearance changes, his stools become formed once more and he may gain considerably in weight. The surgical treats ment is first to separate and close the hole in tho colon and then to proceed to partial gastrectomy either immediately or at a later stage. The dangees of carrying this out at one operation has largelty disappeared with the availability of antibioncy and modern controlled non-toxic anaesthesia.

\section{Recurrent Ulcer after Gastrectomy}

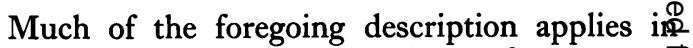
particular to anastomotic ulcer after gastro $\overrightarrow{\overrightarrow{0}}$ enterostomy, still the most usual type of case encountered. However, with the mass change over from short-circuiting operations to gastri? resection in the last decade, a diminished but rea incidence of anastomotic ulcer has become apparent after this operation also. This again applies to duodenal and not to gastric ulcer cases. and for the same reason.

The cause is often to be found in technicat inadequacy of the operation, apart from obvious mistakes such as the use of unabsorbable suture material for the anastomosis. Insufficient removit of the acid-secreting area of the stomach is thor most common error. Another is failure to abolisho entirely the mucosa of the pyloric antrumi although local difficulties may have forced the surgeon to do this. The persistence of the hor monal secretory mechanism in the remaining antral mucosa continues to maintain acid secretion in the gastric remnant after the operation, with $z$ consequent tendency to further ulceration. So much is this the case that when the surgeon feets he has no alternative to dividing the stomach 
proximal to the pylorus, he should later carry out a further operation for the removal of the pylorus and the residual portion of the antrum. The Billroth I method of anastomosis between the stump of the stomach and duodenum also appears to be inadvisable. This technique is excellent for gastric lesions, but appears to carry an increased risk of stomal ulcer if it is used for duodenal cases, even though the ulcer has been completely removed. The addition of an enteroanastomosis between the jejunal loops at the stoma is as unwise in partial gastrectomy as it is with gastro-enterostomy. It should never be necessary. Although the bulk of the acid-secreting mucosa may have been removed, it is asking too much of the resistance of the jejunum to divert the alkaline juices beyond the stoma altogether.

The basic problem remains with us. We understand very little of the essential defence mechanism of the mucosa against erosion. We know that acid peptic activity is the essential factor in the eroding force and that the operation of partial gastrectomy properly performed will, by reducing the scale of the attack, prevent the formation of new ulceration in most cases. This operation, with or without modifications, entails disabilities which in the present state of our knowledge we must accept as a necessary and worthwhile sacrifice to secure freedom from further ulceration. The disquieting reflection, however, is that this sacrifice is really necessary in only a third of the patients, but that all are called upon to make it, because there is yet no practicable preoperative means of selection of cases into those prone to anastomotic ulcer and those not so prone.

\title{
THE TREATMENT OF PYLORIC STENOSIS IN PEPTIC ULCERATIONS
}

\author{
By F. M. Parsons, B.Sc., M.B., Ch.B (Leeds)* \\ Research Fellow iu Urology in the University of Leeds \\ and G. Watkinson, M.D., M.R.C.P. \\ Lecturer in Medicine in the University of Leeds
}

A combination of spasm, oedema and cicatrical deformity in relation to an ulcer near the pyloric ring produces a temporary or permanent retention of gastric contents accompanied by the clinical syndrome of loss of weight, vomiting and dehydration known as pyloric stenosis. The frequent failure of antispasmodic drugs to relieve the condition is in part due to inadequate therapy but is also due to the presence of oedema and scarring.

\section{Physiological Effects}

Obstruction at the pyloric ring leads to increasing retention of gastric contents, gastritis and dilatation of the stomach which eventually leads to vomiting. The reduction in absorption of food causes starvation but it is the extrarenal loss of water, chloride, sodium and potassium, secreted by the gastric mucosa and then vomited, which is

*Aided by grants from the University of Leeds; the Yorkshire Council of the British Empire Cancer Campaign; the Board of Governors, United Leeds Hospitals; and the Leeds Regional Hospital Board. more serious and lethal. As a result of the gastritis there is an increased excretion of gastric mucus which has a high concentration of sodium. This loss of sodium, which leads to a total body deficit, largely determines the extent of the dehydration present. The chloride loss, from the gastric juice, is still, however, in excess of that of the sodium (at least 50 per cent., Gamble, I950) so that an alkalosis results which may be severe enough to cause latent or manifest tetany (Fig. I). Whether this tetany is due to a decrease in ionizable calcium in the extracellular fluid or to other causes has not been clarified. Of equal, if not more, importance is the development of severe renal impairment which occurs in alkalosis. Nazzari (1904) observed calcareous infiltration in the renal tubules in two patients who died as a result of pyloric obstruction. Brown, Eusterman, Hartman and Rowntree (1923) observed at autopsy calcification in the proximal tubules in four patients who died with pyloric stenosis. They tied the pylorus of cats and within 48 hours of 